\title{
Relação entre a capacidade funcional e mobilidade com a prática de atividade física em idosos participantes de uma associação
}

\author{
Relationship between mobility and functional capacity with practice of physical activity \\ in elderly participants of an association
}

Maria Paula Iorio de Moraes ${ }^{a}$, Isabel Almeida Freire Cavalcante de Sousab, Thiago Brasileiro de Vasconcelos ${ }^{\mathrm{a}}$

a Fisioterapeuta. Universidade Federal do Ceará, Universidade Aberta do SUS (Una-SUS), Núcleo de Tecnologias em Educação a Distância em Saúde

Curso de Especialização em Saúde da Pessoa Idosa, Fortaleza, Ceará, Brasil.

b Fonoaudióloga. Escolinha Toca da Criança e Centro de Estudos em Psicologia.

RESUMO Objetivo: Avaliar a relação entre capacidade funcional e mobilidade com a prática de atividade física em idosos participantes de uma associação.

Materiais e Métodos: Estudo do tipo transversal, realizado com um grupo de idosos frequentadores de uma Associação localizada no município de Fortaleza/CE. Os dados foram coletados através de um questionário contendo informações pessoais como sexo, idade, nível de atividade física e a ocorrência de quedas no ano anterior. Em seguida, foram avaliadas a capacidade funcional pelo questionário Brazilian Version of Multidimensional Functional Assessment (BOMFAQ) e a mobilidade por meio do teste Timed Up \& Go (TUG). Para análise dos dados, foram utilizados o teste qui-quadrado e a correlação de Pearson e adotado $p<0,05$ como valor significativo.

Resultados: Participaram do estudo 71 idosos com média de idade de 72,42 47,06 anos, com predomínio do sexo feminino (76,1\%) sendo que 43,7\% afirmaram ter caído nos últimos 12 meses. Não foram encontradas diferenças significativas $(p=0,494)$, quando comparada a prática de atividade física com os escores do BOMFAQ. Já em relação à prática de atividade física e os valores do TUG, foram observadas diferenças significativas $(p=0,022)$, porém, com fraca correlação ( $r=0,310 ; p=0,008)$.

Conclusão: Foi evidenciado que os idosos frequentadores da associação apresentaram déficit para realizar atividades comprometendo a capacidade funcional, no entanto, não foram obsevadas alterações na mobilidade e risco de quedas. Ressalta-se que tais modificações foram mais prevalentes nos idosos que não praticam atividade física.

Palavras-chave: acidentes por quedas; idoso; limitação da mobilidade.

Objective: To evaluate the relationship between mobility and functional capacity with practice of physical activity in elderly participants of an association.

Materials and Methods: Cross-sectional study carried out with a group of elderly people participants of an Association in the city of Fortaleza/CE. Data were collected through a questionnaire containing personal information such as gender, age, level of physical activity and the occurrence of falls in the previous year. Also, functional capacity through the Questionnaire of Multidimensional Functional Assessment (BOMFAQ) and mobility through Timed Up test \& Go (TUG) were evaluated. The chi-square test and Pearson correlation were used for analysis of results and $p<0.05$ was adopted.

Results: The study included 71 older adults with a mean age of $72.42 \pm 7.06$ years, female $(76.1 \%)$ and $43.7 \%$ said that they had fallen in the last 12 months. No significant differences were found $(p=0.494)$ when physical activity was compared to BOMFAQ scores. In relation to physical activity and the TUG values, significant differences were observed $(p=0.022)$, although there was a weak correlation $(r=0.310 ; p=0.008)$.

Conclusion: The study shows that elderly participants of an association have deficits in their ability to perform activities, compromising the functional capacity, although no changes in mobility and risk of fall were observed. Furthermore, it is noteworthy that such modifications were more prevalent in sedentary elderly.

Keywords: accidental falls; elderly; mobility limitation. 


\section{INTRODUÇÃO}

O processo de envelhecimento populacional é um fenômeno mundial que cresce consideravelmente a cada ano. De acordo com a World Health Organization (WHO), projeções indicam que haverá 2 bilhões de idosos acima de 60 anos em 2050'. No Brasil, estima-se que em 2020 haja uma população total de 233,8 milhões, sendo 18,9 milhões os idosos com mais de 65 anos e 3,1 milhões os acima de 80 anos, caracterizando a sexta população mais idosa do mundo em $2025^{2}$.

Estudos indicam que com o avançar da idade, a redução da atividade física gera um declínio funcional dos sistemas cardiovasculares, respiratórios e musculoesqueléticos, limitando as atividades de vida diária, e restringindo ainda mais a atividade física, contribuindo assim, para o progresso do declínio funcional ${ }^{3,4}$.

A diminuição da funcionalidade no idoso favorece várias alterações musculoesqueléticas, como: sarcopenia, alterações no equilíbrio e mobilidade, acarretando risco de quedas nessa população ${ }^{5}$. As quedas constituem uma causa importante a ser estudada nos indivíduos idosos. Cerca de $28-35 \%$ dos idosos com 65 anos ou mais caem pelo menos uma vez por $a^{a} 0^{6}$. Elas causam dois terços dos acidentes na velhice ${ }^{7}$ e são a segunda principal causa de morte por acidentes nos idosos ${ }^{1}$ com grande relevância epidemiológica, social e econômica ${ }^{8}$.

Alguns autores afirmam que cerca de $90 \%$ das fraturas de quadril em indivíduos idosos decorrem das quedas apresentando alta morbidade e mortalidade ${ }^{9}$. Outras complicações que podem ser citadas são: hipoatividade, isolamento social, depressão, institucionalização, redução da qualidade de vida, perda de confiança, dependência nas atividades básicas funcionais e até mesmo o óbito7,10-13.

Ressalta-se que a associação de vários fatores de risco, por exemplo, o déficit da capacidade funcional, equilíbrio e a falta de mobilidade, aumentam ainda mais a possibilidade de quedas do que um só fator isolado ${ }^{14}$ e que há prevalência de quedas tanto em idosos ativos ${ }^{15}$ como naqueles com menor atividade e frágeis ${ }^{14}$.

Diante desse panorama mundial percebe-se cada vez mais a necessidade de estudar os diversos aspectos desse processo para evitar as possíveis alterações que acarretam perda funcional no idoso, e assim, obter um envelhecimento mais ativo e saudável. Nesse sentido, o presente estudo objetivou avaliar a relação entre capacidade funcional e mobilidade com a prática de atividade física em idosos participantes de uma associação.

\section{MATERIAIS E MÉTODOS}

Estudo de campo, do tipo transversal e de natureza quantitativa realizado com um grupo de idosos de diferentes níveis de condicionamento físico, frequentadores da Associação dos Idosos do Mucuripe Otacília Verçosa localizada no município de Fortaleza/CE.

Nessa associação de idosos são realizadas atividades de lazer e outras relacionadas à prevenção e promoção da saúde, tais como: grupos de hipertensos e diabéticos, com palestras, rodas de conversa, ginástica duas vezes na semana e dança de forró uma vez por semana, além de oferecer passeios turísticos, festas comemorativas e um almoço no dia do forró.

Estimou-se a população em torno de 150 idosos. Dessa forma, calculou-se a amostra aleatória de 70 idosos (população finita: erro amostral de 5\%; nível de confiança de 95\%; percentual mínimo de 91\%), sendo, portanto, a quantidade mínima necessária para compor a amostra. Caso algum idoso desistisse de participar do estudo, o mesmo era substituído até atingir o tamanho amostral mínimo. O presente estudo foi aprovado pelo Comitê de Ética em Pesquisa (CEP) da Universidade Federal do Ceará (Protocolo no 660.902). Os idosos que aceitaram participar do estudo assinaram o Termo de Consentimento Livre e Esclarecido.

Como critérios de inclusão, os sujeitos necessitavam ter 60 anos ou mais de idade, desejar participar do estudo, independente do gênero, e tinham que atingir a pontuação mínima do mini exame do estado mental (MEEM) estabelecido por Bertolucci et al. ${ }^{16}$ : 18 pontos para idosos analfabetos; 21 para idosos com 1 a 3 anos de escolaridade; 24 para 4 a 7 anos de escolaridade; 26 para 8 ou mais anos de escolaridade. Foram excluídos os idosos que apresentavam doença neurológica como Parkinson, Acidente Vascular Encefálico e Alzheimer, cegueira, os amputados ou com qualquer tipo de incapacidade motora que influenciasse diretamente na mobilidade. A coleta dos dados foi realizada entre os meses de setembro à novembro de 2014, através da aplicação de um questionário contendo dados socioeconômicos e de saúde (sexo, idade, peso, altura, IMC, escolaridade, atividade física, comorbidades e evento de quedas nos últimos 12 meses), em seguida, foram aplicados os questionários BOMFAQ e TUG.

Para avaliar a capacidade funcional foi utilizado o questionário Brazilian Version of Multidimensional Functional Assessment (BOMFAQ), composto por 15 questões relacionadas às atividades de vida diária (AVD) e atividades instrumentais de vida diária (AIVD) ${ }^{17}$, no qual o idoso responde sobre a sua capacidade atual de realizá-las, classificandoas como "sem dificuldade", "pouca dificuldade" ou "muita dificuldade". O escore total de atividades comprometidas foi feito pela soma das respostas das categorias "pouca" e "muita" dificuldade, sendo que, quanto maior a pontuação, maior o prejuízo na capacidade funcional. 
Para avaliar a mobilidade funcional foi utilizado o Timed Up \& Go (TUG) - com o intuito de avaliar o equilíbrio e a mobilidade funcional por meio do tempo em que o idoso completa a tarefa de se levantar de uma cadeira, andar 3 metros, girar $180^{\circ}$ e voltar a sentar, realizando-a o mais rápido possível sem correr ${ }^{18,19}$.

No presente estudo, foram adotados 12 segundos como tempo normal de realização do TUG para idosos comunitários de acordo com Bischoff et al. ${ }^{20}$. Os mesmos autores determinam valores entre 11-20 segundos para idosos com deficiência ou frágeis, com independência parcial e baixo risco de quedas e valores acima de 20 segundos sugerem idosos com um déficit importante da mobilidade física e alto risco de quedas.

A coleta de dados foi realizada por três pesquisadoras devidamente treinadas, divididas da seguinte forma: a primeira aplicou o MEEM, a segunda o questionário socioeconômico e o TUG e a terceira o BOMFAQ. O TUG foi realizado em um local plano com solo regular ${ }^{20}$. Antes da realização dos testes foram descritas todas as atividades a serem realizadas.

Para análise dos dados foi utilizado o software estatístico SPSS (IBM ${ }^{\circledR}$, New York, Estados Unidos) versão 20, sendo aplicado o teste qui-quadrado e a correlação de Pearson, de acordo com teste de normalidade, e adotando o valor de $p<0,05$ como sendo de significância estatística. Os dados estão apresentados através da média, desvio padrão, valor mínimo (min.) ou máximo (máx.). Particularmente, alguns dados estão descritos em percentual (\%) com o número de observações (n).

\section{RESULTADOS}

Participaram do estudo 71 idosos, que apresentaram uma média no MEEM de 23,11 23,99 [min.=18 e máx. $=30]$, mulheres $(76,1 \%)$, com média de idade de $72,42 \pm 7,06$ [min. $=61$ e máx. $=94]$ anos. Foi evidenciado que a maioria dos idosos possuem de 4 a 6 anos de estudo $(35,2 \%)$, praticam atividade física $(63,4 \%)$ e apresentam índice de massa corporal (IMC) de 26,86 $\pm 4,56$ [min. $=17$ e máx. $=36] \mathrm{kg} / \mathrm{m}^{2}$. Quando questionados se tinham medo de cair, 59,2\% $(n=42)$ informaram que sim. A pesquisa também evidenciou baixa ocorrência de quedas (43\%) (Tabela 1). Em relação à capacidade funcional, $74,6 \%$ dos idosos apresentaram dificuldades em pelo menos uma atividade da vida diária.

A Tabela 2 demonstra que não foram encontradas diferenças significativas ( $p=0,494$; teste qui-quadrado) quando comparada a prática de atividade física com os escores do BOMFAQ.
Tabela 1. Dados socioeconômicos e de saúde dos idosos pesquisados.

\begin{tabular}{|c|c|c|}
\hline Características & $n=71$ & $\%$ \\
\hline $\begin{array}{l}\text { Sexo } \\
\quad \text { Feminino }\end{array}$ & 54 & 76,1 \\
\hline $\begin{array}{l}\text { Idade (anos) } \\
\quad 60-75 \\
76-90 \\
\text { Acima de } 90\end{array}$ & $\begin{array}{c}53 \\
17 \\
1\end{array}$ & $\begin{array}{r}74,6 \\
23,9 \\
1,4\end{array}$ \\
\hline $\begin{array}{l}\text { Escolaridade (anos) } \\
\text { Analfabeto(a) } \\
1-3 \\
4-6 \\
\text { Acima de } 6\end{array}$ & $\begin{array}{l}21 \\
14 \\
25 \\
11\end{array}$ & $\begin{array}{l}29,6 \\
19,7 \\
35,2 \\
15,5\end{array}$ \\
\hline $\begin{array}{l}\text { Atividade Física } \\
\text { Sim } \\
\text { 1-3 dias/semana } \\
\text { 4-7 dias/semana }\end{array}$ & $\begin{array}{l}45 \\
25 \\
20\end{array}$ & $\begin{array}{l}63,4 \\
35,2 \\
28,2\end{array}$ \\
\hline $\begin{array}{l}\text { Tipos de atividades } \\
\text { Caminhada } \\
\text { Forró } \\
\text { Ginástica } \\
\text { Caminhada e Forró } \\
\text { Ginástica e forró } \\
\text { Caminhada e ginástica }\end{array}$ & $\begin{array}{l}16 \\
7 \\
8 \\
8 \\
5 \\
1\end{array}$ & $\begin{array}{c}22,5 \\
9,9 \\
11,3 \\
11,3 \\
7 \\
1,4\end{array}$ \\
\hline $\begin{array}{l}\text { Comorbidades } \\
\text { HAS } \\
\text { DM } \\
\text { ICC } \\
\text { Artrose } \\
\text { HAS e outras } \\
\text { Outras } \\
\text { Nenhuma }\end{array}$ & $\begin{array}{l}7 \\
1 \\
2 \\
5 \\
25 \\
20 \\
11\end{array}$ & $\begin{array}{c}9,9 \\
1,4 \\
2,8 \\
7 \\
35,2 \\
28,2 \\
15,5\end{array}$ \\
\hline $\begin{array}{l}\text { Quedas nos últimos } 12 \text { mese } \\
\text { Nenhuma } \\
1-2 \\
3-4\end{array}$ & $\begin{array}{c}40 \\
26 \\
5\end{array}$ & $\begin{array}{c}56,3 \\
36,7 \\
7\end{array}$ \\
\hline $\begin{array}{l}\text { Hospitalização após a queda } \\
\text { Sim } \\
\text { Não }\end{array}$ & $\begin{array}{c}4 \\
67\end{array}$ & $\begin{array}{c}5,6 \\
94,4\end{array}$ \\
\hline
\end{tabular}

HAS: hipertensãoarterial sistêmica; DM: diabetes mellitus; ICC: insuficiência cardíacacongestiva; Outras: alergia, hemorroida, glaucoma. Frequência de sujeitos (n) e frequência relativa percentual $(\%)$

Tabela 2. Comparação entre os escores do BOMFAQ em relação à prática de atividade física pelos idosos.

\begin{tabular}{|c|c|c|c|c|}
\hline \multirow{2}{*}{\multicolumn{2}{|c|}{ BOMFAQ }} & \multicolumn{2}{|c|}{ Atividade Física } & \multirow{2}{*}{ Total } \\
\hline & & Sim & Não & \\
\hline \multirow{2}{*}{1} & $\mathbf{n}$ & 14 & 4 & 18 \\
\hline & $\%$ & $77,8 \%$ & $22,2 \%$ & $100,0 \%$ \\
\hline \multirow{2}{*}{2} & $\mathbf{n}$ & 15 & 12 & 27 \\
\hline & $\%$ & $55,6 \%$ & $44,4 \%$ & $100,0 \%$ \\
\hline \multirow{2}{*}{3} & $\mathbf{n}$ & 14 & 9 & 23 \\
\hline & $\%$ & $60,9 \%$ & $39,1 \%$ & $100,0 \%$ \\
\hline \multirow{2}{*}{4} & $\mathbf{n}$ & 2 & 1 & 3 \\
\hline & $\%$ & $66,7 \%$ & $33,3 \%$ & $100,0 \%$ \\
\hline \multirow{2}{*}{ Total } & $\mathbf{n}$ & 45 & 26 & 71 \\
\hline & $\%$ & $63,4 \%$ & $36,6 \%$ & $100,0 \%$ \\
\hline
\end{tabular}

Escores do BOMFAQ: 1 = sem dificuldade; 2 = dificuldade em 1 a 3 atividades; 3 = dificuldade em 4 a 6 atividades e $4=$ dificuldade em 7 ou mais atividades. $p=0,494$; teste qui-quadrado. Frequência de sujeitos (n) e frequência relativa percentual (\%). 
Já em relação aos dados encontrados pelo TUG, $80 \%$ dos idosos não apresentaram comprometimento da mobilidade e risco de quedas. Quando comparado o TUG com a prática de atividade física, foram observadas diferenças significativas ( $p=0,022$; teste qui-quadrado) (Figura1). No entanto, houve fraca correlação ( $r=0,310 ; p=0,008$ - correlação de Pearson).

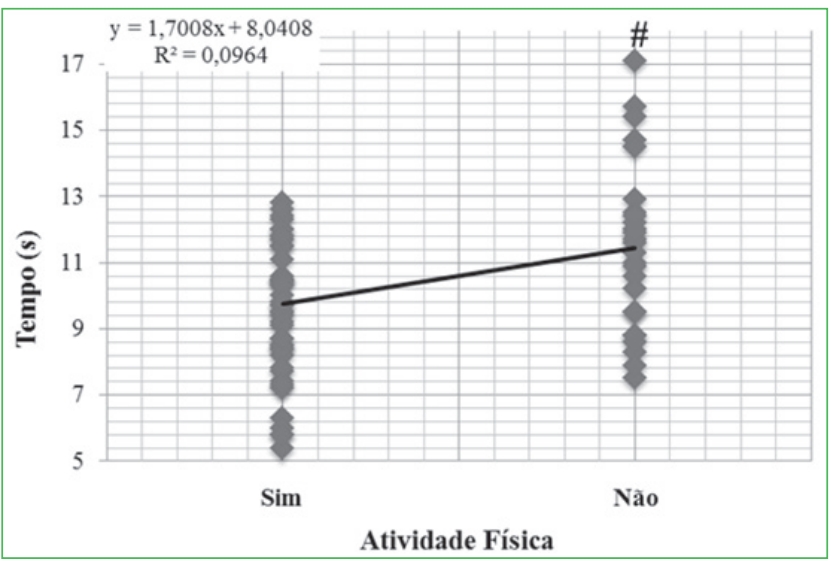

Figura 1. Comparação entre os dados de mobilidade em relação à prática de atividade física de idosos. TUG expresso em segundos. 1 = Normal $(12$ segundos); 2 = Baixo risco de queda (12,1 a 20 segundos) e $3=$ Alto risco de queda (acima de 20 segundos). \#p=0,022; teste qui-quadrado.

\section{DISCUSSÃO}

O presente estudo apontou uma diferença significativa entre os idosos que frequentam a associação, evidenciando que a prática regular de atividades físicas está associada a uma melhor mobilidade funcional e menor risco de quedas. No entanto, não foi observada diferença significativa quando comparada a capacidade funcional com a prática de atividade física.

A maior parte da amostra foi composta por mulheres ativas eutróficas. Foi encontrada maior prevalência de hipertensão, doença frequentemente encontrada na população idosa ${ }^{5,21}$. Em relação à escolaridade os dados revelaram que os idosos tiveram um acesso limitado ao ensino escolar ${ }^{22-23}$.

Em relação à capacidade funcional, os dados obtidos pelo BOMFAQ evidenciaram que a maioria $(74,6 \%)$ dos idosos pesquisados apresentaram dificuldade em pelo menos uma atividade da vida diária. Esses dados são superiores aos encontrados por Couto e Perracini ${ }^{6}$, que também analisaram através do BOMFAQ a capacidade funcional de idosos ativos e concluíram que $54 \%$ encontraram alguma dificuldade nas AVDs.

Esta ausência de significância estatística entre os dados do BOMFAQ e a atividade física, demonstra não ser possível afirmar que há uma relação direta entre essas duas variáveis. Isto pode ser atribuído à homogeneidade da amostra, uma vez que, embora nem todos os idosos pratiquem uma atividade física regular, eles apresentam uma vida ativa, já que frequentam uma associação e desfrutam de uma convivência social, o que difere de um perfil propriamente sedentário. No entanto, não se sabe ao certo o nível de atividade que influenciou esses dados ${ }^{24}$.

A literatura indica que os idosos ativos apresentam menor risco de fragilização e declínio funcional, fatores esses que podem predispor as quedas. Outro fator que pode influenciar a ocorrência de quedas é o medo de cair. Cumming et al. ${ }^{25}$ indicam que idosos com medo de cair possuem baixa autoconfiança em realizar atividades do dia-a-dia e tendem a ter um comprometimento progressivo em sua capacidade funcional ao longo do tempo. Esse medo está associado ao risco de quedas no futuro, mesmo em pessoas que não sofreram queda.

Alguns autores acrescentam que a prática regular de exercício físico pode ser considerada um fator protetor para quedas, pois promove um aumento da força e resistência muscular, melhora o controle motor e o equilíbrio corporal ${ }^{26}$, possibilitando executar as atividades do cotidiano com maior competência ${ }^{27}$.

O estudo de Lessa ${ }^{5}$ indica que conforme a faixa etária aumenta, há uma redução da massa muscular, e consequentemente, da força, podendo interferir na mobilidade e no risco de quedas. Além disso, esses idosos com o passar do tempo tendem a diminuir suas atividades físicas, diminuindo os fatores de risco externos e se expondo mais no ambiente doméstico.

Neste contexto, foi utilizado o TUG para avaliar a mobilidade dos idosos e predizer o risco de quedas, sendo observado que os idosos apresentaram um desempenho normal no teste, ou seja, valores inferiores a 12 segundos. Dado que corrobora com o estudo de Couto e Perracini ${ }^{6}$, em que analisaram o perfil de idosos ativos com história de quedas. Em uma amostra de 150 idosos, 89,3\% obtiveram um valor inferior a 12 segundos no TUG, evidenciando uma boa mobilidade.

Diversos estudos ${ }^{6,28-30}$ associam o TUG e histórico de quedas. O TUG é um dos testes mais usados para avaliação da mobilidade, equilíbrio e risco de quedas em idosos ${ }^{19,31}$. É uma medida prática que não utiliza equipamento especial ou treinamento, sendo por isso um instrumento de fácil aplicação no ambiente da atenção básica ${ }^{18}$.

O estudo de Whitney et al. ${ }^{29}$ indicam o TUG como um bom preditor de quedas, no qual identificaram o TUG como uma ferramenta de triagem simples para identificar indivíduos com risco de queda. Os autores concluíram que o instrumento associado a um teste simples de cognição pode ser usado para agilizar referências em uma população 
de alto risco, permitindo o uso mais eficiente dos recursos disponíveis na prática clínica.

No entanto, há estudos que apresentam limitações do TUG como o de Thrane et al..$^{30}$, que avaliaram a associação entre TUG e histórico de quedas em idosos residentes na comunidade. Fez-se um estudo retrospectivo, de base populacional, observacional, realizado com 414 homens e 560 mulheres. Foram registrados o tempo do TUG e as quedas durante os 12 meses anteriores. A pesquisa abrangeu a influência de covariáveis de idade, sexo, história médica e problemas de mobilidade relacionados com a saúde. Os resultados da pesquisa apontaram que o TUG tem uma capacidade limitada de classificar os idosos propensos a quedas.

Já Steffen et al. ${ }^{28}$, utilizaram uma série de instrumentos incluindo o TUG para avaliar a mobilidade física e a propensão a quedas em idosos. O estudo apontou a capacidade de medição do TUG com a ressalva de que os dados obtidos com o instrumento devem ser interpretados sob a influência da variável idade.

Como a amostra deste estudo foi composta por uma maioria de idosos ativos, era de se esperar que não houvesse uma redução significativa da mobilidade conforme os estudos que apontam os fatores de risco intrínsecos, como fraqueza muscular, distúrbios do equilíbrio corporal e da marcha, os mais determinantes para quedas em idosos frágeis. Já para os idosos ativos, a exposição aos fatores de risco ambientais e as tarefas que exigem uma maior demanda sobre o equilíbrio corporal seriam considerados fatores de risco de maior relevância para quedas ${ }^{6}$.

Dentre as limitações do estudo podemos inferir o tamanho amostral, os diferentes níveis de condicionamento físico e a falta de descrição detalhada da atividade física realizada pelos mesmos, no entanto, destaca-se que o objetivo do estudo foi atingido.

Foi evidenciado que os idosos frequentadores da associação apresentaram déficit para realizar atividades comprometendo a capacidade funcional, no entanto, não foram obsevadas alterações na mobilidade e risco de quedas. Ressalta-se que tais modificações foram mais prevalentes nos idosos que não praticam atividade física. Por fim, ainda é incerto qual nível de atividade física está atrelado ao maior risco de quedas; no entanto, as atividades desenvolvidas em grupos de idosos ou associações, podem contribuir para a saúde e favorecer a convivência social, proporcionando melhor qualidade de vida.

\section{REFERÊNCIAS}

1. World Health Organization. WHO global report on falls prevention in older age. [acesso em 2014 ago. 30]. Disponível em: http://www. who.int/ageing/publications/Falls_prevention7March.pdf?ua =1
2. Bechara FT, Santos SMS. Efetividade de um programa fisioterapêutico para treino de equilíbrio em idosos. Rev Saúde Pesq. 2008; 1(1):15-20.

3. Ferreira MT, Matsudo SMM, Ribeiro MCSA, Ramos LRR. Healthrelated factors correlate with behavior trends in physical activity level in old age: longitudinal results from a population in Sao Paulo, Brazil. BMC Public Health. 2010;10:690. http://dx.doi. org/10.1186/1471-2458-10-690

4. Lang PO, Michel JP, Zekry D. Frailty syndrome: a transitional state in a dynamic process. Gerontology. 2009;55(5):539-49. http://dx.doi. org/10.1159/000211949

5. Lessa I. O Adulto brasileiro e as doenças da modernidade: epidemiologia das doenças crônicas não transmissíveis. Ciênc Saúde Coletiva 2004;9(4):931-43. http://dx.doi.org/10.1590/S141381232004000400014

6. Couto FBDE, Perracini MR. Análise multifatorial do perfil de idosos ativos com história de quedas. Rev Bras Geriatr Gerontol. 2012;15(4):693-706. http://dx.doi.org/10.1590/S1809-9823201 2000400010

7. Nachreiner NM, Findorff MJ, Wyman JF, Mccarthy TC. Circumstances and consequences of falls in community-dwelling older women. J Womens Health (Larchmt). 2007;16(10):1437-46. http://dx.doi. org/10.1089/jwh.2006.0245

8. Rebelatto JR, Castro AP, Sako FK, Aurichio TR. Equilíbrio estático e dinâmico em indivíduos senescentes e o índice de massa corporal. Fisioter Mov. 2008;21(3):69-75.

9. Simoceli L, Bittar RMS, Bottino MA, Bento RF. Perfil diagnóstico do idoso portador de desequilíbrio corporal: resultados preliminares. Rev Bras Otorrinolaringol. 2003;69(6):772-7. http://dx.doi. org/10.1590/S0034-72992003000600008

10. Perracini MR, Ramos LR. Fatores associados a quedas em uma coorte de idosos residentes na comunidade. Rev Saúde Pública. 2002;36(6):709-16. http://dx.doi.org/10.1590/S003489102002000700008

11. Skelton DA, Beyer, N. Exercise and injury prevention in older people. Scand J Med Sci Sports. 2003;13(1):77-85. http://dx.doi. org/10.1034/j.1600-0838.2003.00300.x

12. Inouye SK, Studenski S, Tinetti ME, Kuchel GA. Geriatric syndromes: clinical, research and policy implications of a core geriatric concept. J Am Geriatr Soc. 2007;55(5):780-91. http://dx.doi.org/10.1111/ j.1532-5415.2007.01156.x

13. Lojudice DC, Laprega MR, Gardezani PM, Vidal P. Equilíbrio e marcha de idosos residentes em instituições asilares do município de Catanduva, SP. Rev Bras Geriatr Gerontol. 2008;11(2):181-9.

14. Tinetti ME, Kumar C. The patient who falls: "It's always a tradeoff". JAMA. 2010;303(3):258-66. http://dx.doi.org/10.1001/ jama.2009.2024

15. Chan BK, Marshall LM, Winters KM, Faulkner KA, Schwartz AV, Orwoll ES. Incident fall risk and physical activity and physical performance among older men: the Osteoporotic Fractures in Men Study. Am J Epidemiol. 2007;165(6):696-703. http://dx.doi. org/10.1093/aje/kwk050

16. Bertolucci PHF, Brucki SMD, Campacci SR, Juliano Y. O MiniExame do Estado Mental em uma População Geral: Impacto da Escolaridade. Arq Neuro-Psiquiatr. 1994;52(1):1-7. http://dx.doi. org/10.1590/S0004-282X1994000100001 
17. Ramos LR, Toniolo JN, Cendoroglo MS, Garcia JT, Najas MS, Perracini M, Paola CR, Santos FC, Bilton T, Ebel SJ, Macedo MBM, Almada CMF, Nasri F, Miranda RD, Gonçalves M, Santos ALP, Fraietta R, Vivacqua IN, Alves MLM, Tudisco ES. Two-year followup study of elderly residents in S. Paulo, Brazil: methodology and preliminary results. Rev Saúde Pública. 1998;32(5):397-407. http://dx.doi. org/10.1590/S0034-89101998000500001

18. Podsiadlo D, Richardson S. The timed "Up \& Go": a test of basic functional mobility for frail elderly pearsons. J Am Geriatr Soc. 1991;39(2):142-8. http://dx.doi.org/10.1111/j.1532-5415.1991. tb01616.x

19. Shimada H, Suzukaw M, Tiedemann A, Kobayashi K, Yoshida H, Suzuki T. Which neuromuscular or cognitive test is the optimal screening tool to predict falls in frail community-dwelling older people? Gerontology. 2009;55(5):532-8. http://dx.doi. org/10.1159/000236364

20. Bischoff HA, Stähelin HB, Monsch AU, Iversen MD, Weyh A, Von Dechend M, Akos R, Conzelmann M, Dick W, Theiler R. Identifying a cut-off point for normal mobility: A comparison of the timed 'up and go' test in community-dwelling and institutionalised elderly women. Age Ageing. 2003;32(3):315-20. http://dx.doi.org/10.1093/ ageing/32.3.315

21. Carneiro FR, Brasileiro IC, Vasconcelos TB, Arruda VP, Florêncio RS, Moreira TMM. Independência funcional de idosas residentes em instituições de longa permanência. Acta Fisiátr. 2012;19(3):156-60. http://dx.doi.org/10.5935/0104-7795.20120024

22. Braga DKAP, Domiciano BR, Santos MPA, Vasconcelos TB, Macena RHM. Relação entre idade e capacidade funcional de idosas residentes em instituições de longa permanência em uma capital do nordeste brasileiro. J Health Biol Sci. 2014;2(4):197-201. http:// dx.doi.org/10.12662/2317-3076jhbs.v2i4.82.p197-201.2014

23. Domiciano BR, Braga DKA Peixoto, Silva PN, Santos MPA, Vasconcelos TB, Macena RHM. Cognitive function of elderly residents in long-term institutions: effects of a physiotherapy program. Rev Bras Geriatr Gerontol. 2016;19(1):57-70. http:// dx.doi.org/10.1590/1809-9823.2016.14137

24. Peeters GM, Van Schoor NM, Pluijm SM, Deeg DJ, Lips P. Is there a U-shaped association between physical activity and falling in older persons? Osteoporos Int. 2010;21(7):1189-95. http://dx.doi. org/10.1007/s00198-009-1053-4

25. Cumming RG, Salked G, Thomas M, Szonyi G. Prospective study of the impact of fear of falling on activities of daily living, SF-36 scores, and nursing home admission. J Gerontol A Biol Sci Med Sci. 2000;55(5):M299-305. http://dx.doi.org/10.1093/ gerona/55.5.M299

26. Rose DJ. Preventing falls among older adults: no "one size suits all" intervention strategy. J Rehabil Res Dev. 2008;45(8):1153-66. http:// dx.doi.org/10.1682/JRRD.2008.03.0041

27. Ricci NA, Gonçalves DFF, Coimbra IB, Coimbra AMV. Fatores associados ao histórico de quedas de idosos assistidos pelo programa de saúde da família. Saúde Soc. 2010;19(4):898-909. http://dx.doi. org/10.1590/S0104-12902010000400016

28. Steffen TM, Hacker TA, Mollinger L. Age- and Gender-Related Test Performance in Community-Dwelling Elderly People: Six-Minute Walk Test, Berg Balance Scale, Timed Up \& Go Test, and gait Speeds. Phys Ther. 2002;82(2):128-37.

29. Whitney JC, Lord SR, Close JC. Streamlining assessment and intervention in a falls clinic using the Timed Up and Go Test and Physiological Profile Assessments. Age Ageing. 2005;34(6):567-71. http://dx.doi.org/10.1093/ageing/afi178

30. Thrane G, Joakimsen RM, Thornquist E. The association between timed up and go test and history of falls: the Tromsø study. BMC Geriatr. 2007;7:1. http://dx.doi.org/10.1186/1471-2318-7-1

31. Ayan C, Cancela JM, Gutiérrez A, Prieto I. Influence of the cognitive impairment level on the performance of the Timed "Up \& Go" Test (TUG) in elderly institutionalized people. Arch Gerontol Geriatr. 2013;56(1):44-9. http://dx.doi.org/10.1016/j.archger.2012.06.002 